\section{A New Embedding Medium for Electron Microscopy}

THE introduction of $n$-butyl and methyl methacrylates as embedding media ${ }^{1}$ constituted an important development in the preparation of ultra-thin sections of biological material; but their use is attended by various major difficulties. Shrinkage occurs on polymerization, hard objects are not penetrated by the monomer so that the plastic shrinks away and does not provide sufficient support, and sometimes there is an unaccountable appearance of bubbles around the specimen. Moreover, the poly. merization process is not uniform and sometimes causes severe damage. This is perhaps most strikingly seen in bacteria where often only empty remnants of cell walls remain.

Attempts have been made to minimize these distorting effects by modifying the methacrylate procedure $^{2}$, but only with partial success. The substitution of a different embedding medium seems desirable. The possible usefulness of the epoxy resins for the preparation of ultra-thin sections of bacteria has already been indicated ${ }^{3}$.

We have examined a series of epoxy resins, supplied under the name of 'Araldite'*, for their suitability as embedding media. On the addition of a hardener, which is a liquid anhydride, the resin sets slowly without shrinkage to form a light-gold block. The viscosity of the mixture and the hardness of the final block can be controlled with a plasticizer, dibutyl phthalate, and the rate of hardening can be varied with an amine accelerator. By a suitable variation of these components it is possible to produce an embedding medium with the properties required for electron microscopy. Slightly different combinations will be found convenient for different types of specimen; one such combination is described here.

The components were measured into a graduated cylinder warmed to $48^{\circ} \mathrm{C}$. to facilitate drainage and were mixed in the following order:

$\begin{array}{lr}\text { 'Araldite' } M & 100 \mathrm{ml} \\ \text { Hardener 964 B } & 100 \mathrm{ml} . \\ \text { Plasticizer } & 10 \mathrm{ml} . \\ \text { Accelerator } 964 \mathrm{C} & 3 \mathrm{ml} .\end{array}$

Although the mixture can be stored at $s^{\circ} \mathrm{C}$. for several days we found it convenient to prepare it in quantities one-tenth of those indicated. After stirring, a clear, golden liquid resulted which was more viscous than methacrylate monomers; but the viscosity could be reduced by holding the solution at $48^{\circ} \mathrm{C}$. and infiltrating the specimens at this temperature.

In the preliminary trials the 'Araldite' mixture has been used for embedding bacteria, fragments of lung and mature hair fibres. After fixation and dehydration the specimens were soaked for $1-2 \mathrm{hr}$. in a $50 / 50$ mixture of alcohol and 'Araldite'. They were then transferred to pure 'Araldite' for 2-3 hr. at $48^{\circ} \mathrm{C}$., and finally placed in No. 00 gelatin capsules with fresh 'Araldite'. Curing for $48 \mathrm{hr}$. yielded a light-gold block with a hardness comparable with methacrylate.

'The 'Araldite' had excellent cutting characteristics ; sections were floated on to 20 per cent acetone-water, mounted on carbon films and examined in the electron microscope. Their electron density was similar to

* Trade name for epoxy resins developed by Messrs. Ciba, Ltd., Basle, and made in Great Britain by Aero Research, Ltd., Duxford,
Cambridge, from whom all the components described are available. methacrylate. As had been hoped, bacteria were intact, and hair fibres were sufficiently well supported to allow thin sections to be cut and details of internal structure to be revealed.

It is considered that such an alternative embedding medium will be valuable for studies of tissue fine structure; but the resin must be tested on many different types of specimen before its potentialities can be fully assessed.

We are indebted to Dr. V. E. Cosslett and Mr. R. W. Horne, of the Cavendish Laboratory, Cambridge, for providing electron microscope facilities. One of us (G. E. R.) would like to thank the Commonwealth Scientific and Industrial Research Organization, Australia, for a studentship.

Strangeways Research Laboratory,

Audrey M. Glauert* Cambridge.

G. E. Rogers

Department of Anatomy, Cambridge.

R. H. GLAUERT

Aero Research, Ltd.,

Duxford, Cambridge.

* Sir Halley Stewart Research Fellow.

${ }^{1}$ Newman, S. B., Borysko, E., and Swerdlow, M., J. Res. Nat. Bur. Stand., 43, 183 (1949).

${ }^{2}$ Borysko, E., and Sapranauskas, P., Johns Hopkins Hosp. Bull., 95, $68(1954)$.

${ }^{3}$ Maaløe, O., and Birch-Andersen, A., Sixth Symp. Soc. Gen. Microbiol. "Bacterial Anatomy", 261 (1956).

\section{Re-appraisal of the Evidence for the Metabolic Conversion of Vasopressin into a Less Active Derivative}

THE intermediary metabolism of peptide hormones is unknown, and it is therefore of great interest that Dicker and Greenbaum ${ }^{1}$ have recently suggested "that both kidney and liver slices transform vasopressin into substances of lower antidiuretic activity". They reached this conclusion because they found that the activity of 'Pitressin', when incubated with rat kidney slices, decreased until 20 per cent of the initial antidiuretic potency remained; thereafter, there was no further inactivation. When the resultant supernatant from such an incubation mixture was added to fresh kidney slices its activity remained constant although it could be completely abolished by liver slices. Thus, the property by which Dicker and Greenbaum distinguish between vasopressin and the product of its metabolism is that the latter resists inactivation by kidney slices while vasopressin does not.

The results of in vitro experiments do not necessarily apply to the fate of vasopressin in vivo, but Dicker and Greenbaum attempted to show that, following intra. venous infusion of vasopressin into rats anæsthetized with ethanol, the metabolic derivative of vasopressin is excreted in urine. After infusing large amounts of vasopressin they found that the antidiuretic activity excreted in urine was within the range found in previous investigations $(3 \cdot 1-22 \cdot 0$ per cent of the dose infused ${ }^{2}$ ). However, when urine containing this excreted activity was infused into another rat, they claim that 48-59 per cent of the dose now appeared in the urine. The explanation offered is that liver and kidneys in vivo treat vasopressin and the antidiuretic activity in urine as do slices of these organs. 